\title{
CARCINOMA DE CUELLO UTERINO REVISION. Y MANEJO
}

\section{Hospital Regional Federico Lleras - Ibagué}

Trabajo presentado al III Congreso

Colombiano de Cancerología

Junio de 1.977

\section{Doctor Jaime Rengifo Pardo*}

Señores Oscar Rusinsky, Aída Vargas, María de la Luz Valencia, José Moreno, Internos Rotatorios.

Este trabajo no muestra nada nuevo, pero significa el estudio y revisión de los casos de carcinoma del cuello uterino en los dos primeros años de funcionamiento del Hospital Regional Federico Lleras de Ibagué.

\section{MATERIAL Y METODOS ..}

Se analizan 80 casos de carcinoma

* Jefe Departamento Ginecoobstetricia Hospital Federico Lieras. del cuello vistos en el Departamento de Obstetricia y Ginecología del Hospital Regional Federico Lleras de Ibagué, en el período comprendido entre el 1 o de agosto de 1.974 y el 31 de Julio de 1.976. Aunque solamente los carcinomas in situ, y los microinvasivos fueron tratados por nosotros, se presentan los casos de carcinomas invasivos previamente diagnosticados, fueron enviados al Instituto Nacional de Cancerología, salvo los considerados intratables que no fueron remiti. dos.

\section{DISTRIBUCION}

Gráfica № 1

HOSPITAL FEDERICO LLERAS ACOSTA

CARCINOMA CUELLO UTERINO Agosto 74 - Junio 76 DISTRIBUCION

\begin{tabular}{|l|r|}
\hline \multicolumn{2}{|l|}{} \\
\hline TOTAL INGRESOS HOSPITALARIOS & 13.207 \\
\hline TOTAL INGRESOS OBSTETRICOS & $5.806:=1.37 \%$ \\
\hline TOTAL CASOS CA CUELLO UTERINO & $80=1.37 \%$ \\
\hline IN SITU & $46=57.5 \%$ \\
\hline INVASIVO & $34=42.5 \%$ \\
\hline
\end{tabular}


El total de ingresos hospitalarios en ese período fue de 13.207. El total de ingresos al departamento de ginecoobstetricia fue de 5.806, sobre este total se presentaron 80 carcinomas de cuello, para un $1,37 \%$ de los ingresos en ginecoobstetricia.

Así mismo se discriminaron así.

Carcinoma in situ 46 casos $57,5 \%$ Carcinoma invasivo 34 casos $42,5 \%$

EDAD

Gráfica Nọ 2

HOSPITAL FEDERICO LLERAS ACOSTA

CARCINOMA CUELLO UTERINO Agosto 74 - Junio 76 EDAD

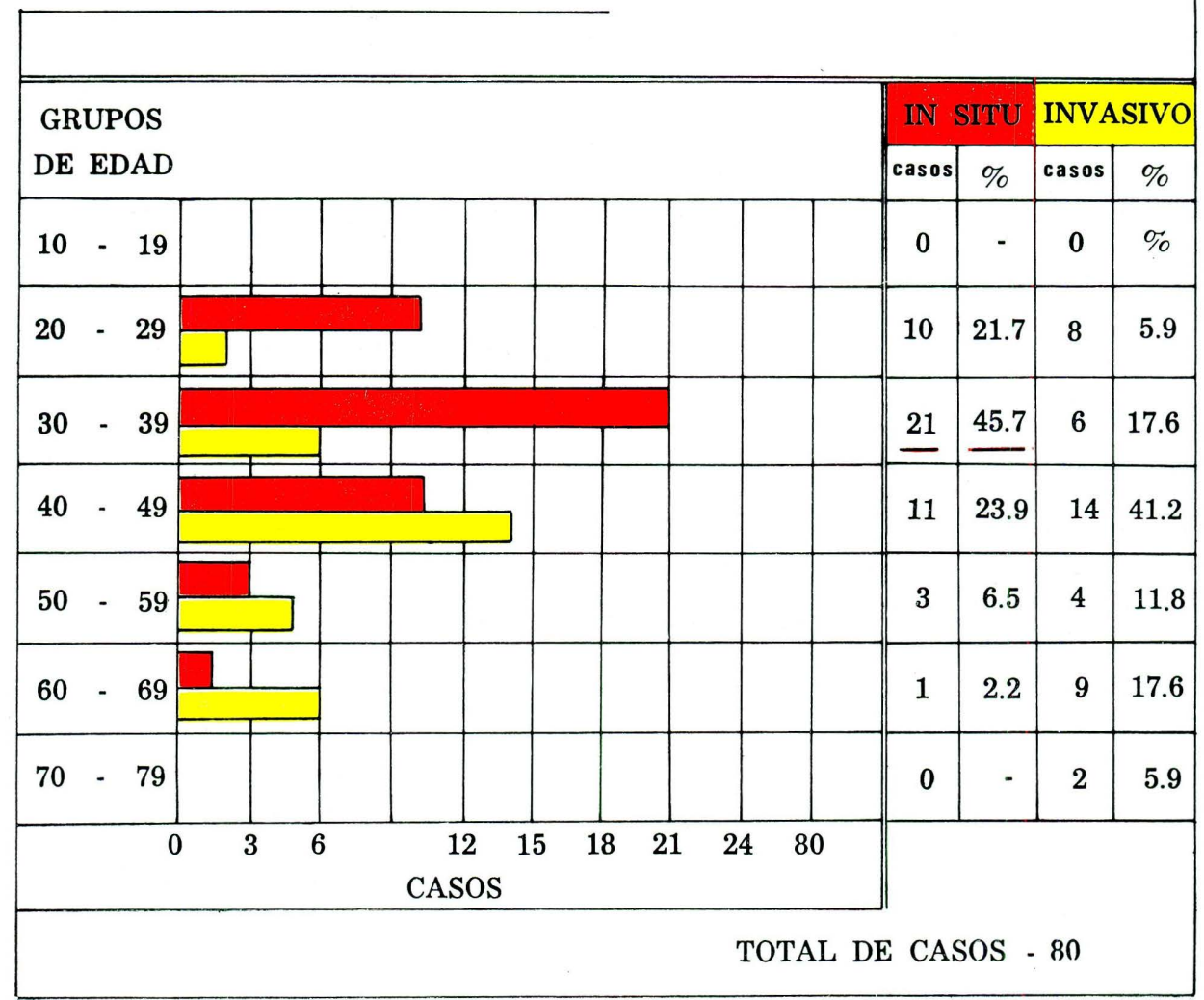

No se presentaron casos en menores de 20 años. La mayor incidencia para carcinoma in situ fue en el grupo de
30 a 39 años con un $45,7 \%$. Para el carcinoma invasivo el grupo de 40 a 49 años con $41,2 \%$. 
Vol. XXVIII

PARIDAD

Gráfica № 3

HOSPITAL FEDERICO LLERAS ACOSTA

CARCINOMA CUELLO UTERINO Agosto 74 - Junio 76

PARIDAD

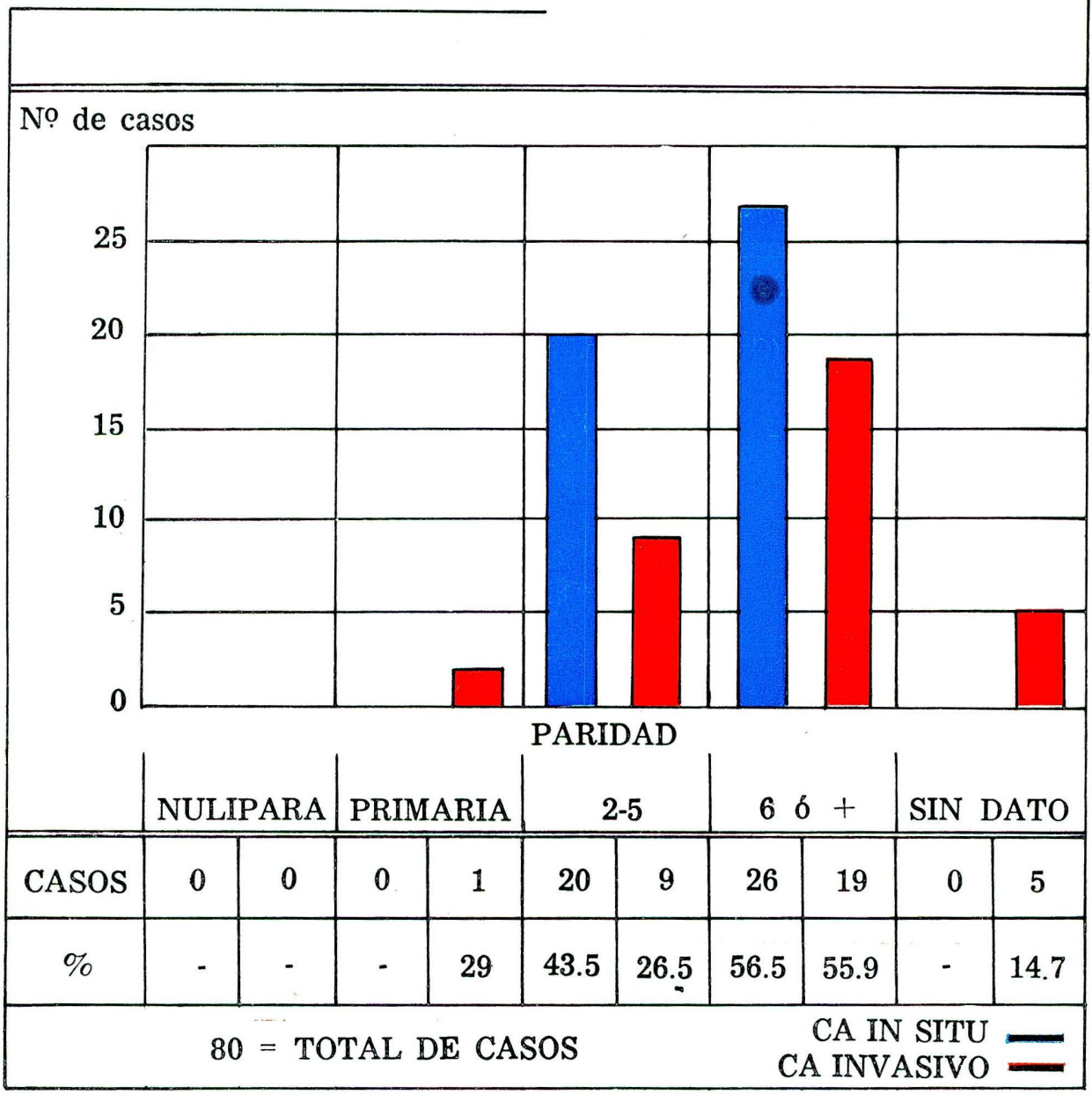

No encontramos en nulíparas. En primíparas solo se presentó un caso de carcinoma invasivo. En cambio la in. cidencia fue más alta en las multípa- ras, con mayores índices en los grupos de 6 o más hijos, que en los grupos de 2 a 5 hijos. 
PROCEDENCIA

Gráfica No 4

HOSPITAL FEDERICO LLERAS ACOSTA

CARCINOMA CUELLO UTERINO Agosto 74 - Junio 76 PROCEDENCIA

\begin{tabular}{|c||c|c|c|c|}
\hline \multicolumn{1}{|c||}{} & \multicolumn{2}{c|}{ IN SITU } & \multicolumn{2}{c|}{ INVASIVO } \\
\hline \hline URBANA & 32 & 69.6 & 20 & 58.8 \\
\hline RURAL & 14 & 30.4 & 14 & 41.2 \\
\hline
\end{tabular}

En relación a la procedencia los invasivo respectivamente, mientras la mayores porcentajes corresponden a rural tiene $30.4 \%$ y $41.2 \%$ en casos la zona urbana, $69,6 \%$ y $58.8 \%$ in situ $\quad$ in situ e invasivo.

\section{ASPECTO CLINICO (Gráficas Nos.5 y 6)}

Gráfica № 5

HOSPITAL FEDERICO LLERAS ACOSTA

CARCINOMA CUELLO UTERINO Agosto 74 - Junio 76 ASPECTO CLINICO DEL CUELLO

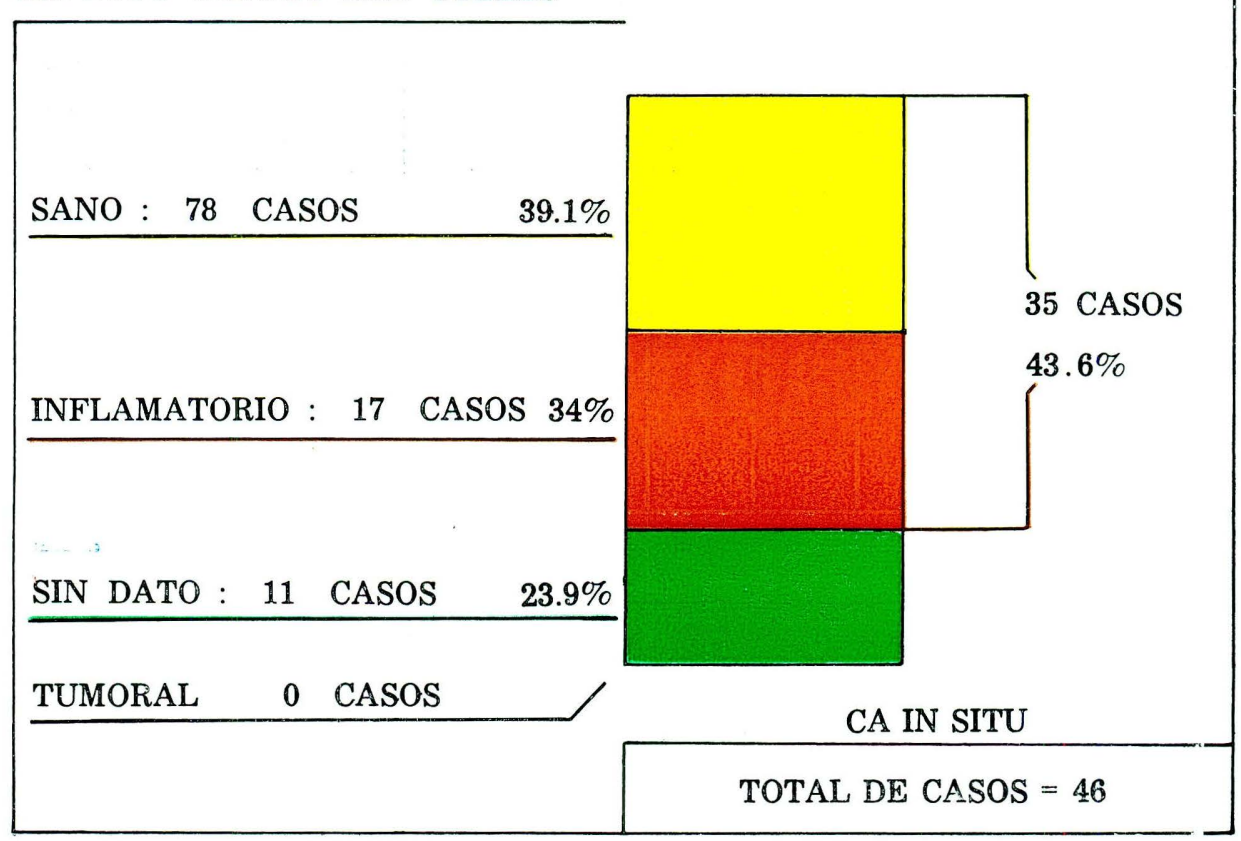


Gráfica Nọ 6

HOSPITAL FEDERICO LLERAS ACOSTA

CARCINOMA CUELLO UTERINO Agosto 74 - Junio 76

ASPECTO CLINICO DEL CUELLO

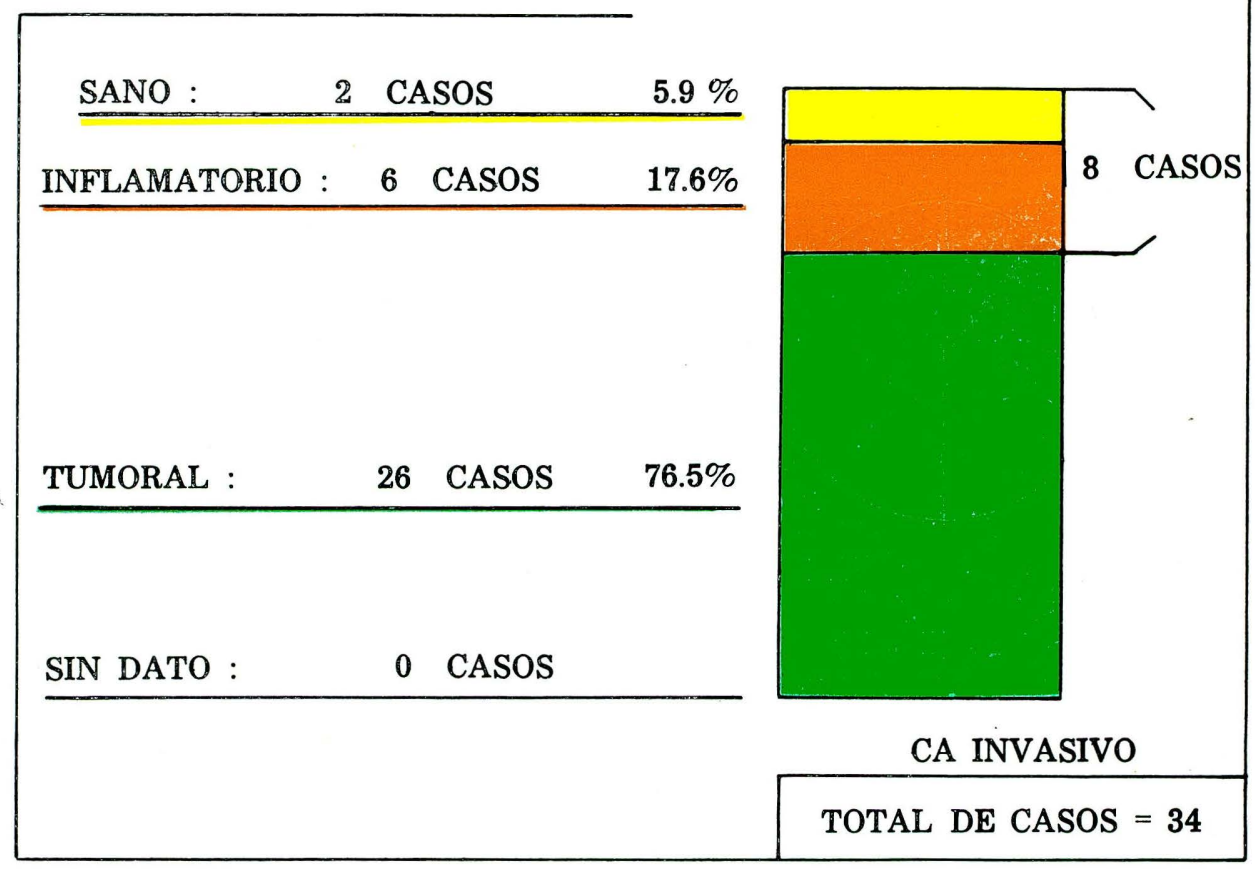

En el carcinoma in situ, encontra$17,6 \%$ y como cuello sano un $5,9 \%$ mos un $39,1 \%$ de cuellos sanos, un $34 \%$ de cuellos inflamatorios y un $23,9 \%$ sin dato claro en historia clínica de admisión, pero no se encontró ningún caso reportado como tumorai.

En tanto, en el carcinoma invasivo se reportaron como tumorales un $76,5 \%$ de los casos, inflamatorios un (casos posteriormente reportados como microinvasivos).

\section{METODOS DIAGNOSTICOS}

Se utilizaron la citología y la biopsia. No hay estudios colposcópicos por carecer el Hospital de colposcopia. 


\section{CITOLOGIA}

Gráfica Nọ 7

HOSPITAL FEDERICO LLERAS ACOSTA

CARCINOMA DE CUELLO UTERINO Agosto 74 - Junio 76

DIAGNOSTICO

\begin{tabular}{|c|c|}
\hline CITOLOGIA VAGINAL & $50 \%$ \\
\hline Negativa (I - II) 3 CASOS & Negativa $(\mathrm{I}-\mathrm{II})$ \\
\hline Dudosa (III ) 18 CASOS & Dudosa $\quad$ (III ) $\quad 3$ CASOS \\
\hline Positiva (IV - V) 21 CASOS & Positiva (IV - V) \\
\hline TOTAL DE 42 CASOS & TOTAL DE 6 CASOS \\
\hline $\begin{array}{ccc}\text { NO SE PRACTICO EN } & \text { PRAS } \\
4 & \text { CASOS }\end{array}$ & NO SE PRACTICO EN 28 CASOS \\
\hline
\end{tabular}

Se practicó en 42 de los 46 casos, dudosa en 18, y positiva en 21. En cuatro casos no se practicó porque las pacientes venían remitidas de Profamilia con biopsia positiva.
En los 36 casos de carcinoma inva. sivo solo se practicó en 6, tres repor. tados como dudosa y 3 como positiva. En los demás casos se fue directamente a biopsia. 
BIOPSIA (Gráfica Nọ 8)

La biopsia fue reportada como car. cinoma in situ en 45 casos, 97,8\% y zomo displasia-severa en un caso $2.2 \%$

En el carcinoma invasivo se reportaron en 31 con $91,2 \%$ en tres casos no se practicó biopsia.

\section{TRATAMIENTO}

En los 46 casos presentados se efec. tuó conización o histerectomía ampliada. 18 conizaciones y 28 histerectomías 40 y $60 \%$ respectivamente.

De los 18 conos fueron reportados 15 como carcinoma in situ y tres co mo displasia-severa (gráfica 9). De las 28 histerectomías ampliadas 26 fueron reportadas como carcinoma in situ y dos como carcinoma microinvasivo.

De los casos de carcinomas invasi. vos 23 fueron enviados al Instituto Nacional de Cancerología, y los 9 res. tantes fueron considerados intrata. bles.
Gráfica Nọ 8

HOSPITAL FEDERICO LLERAS ACOSTA

CARCINOMA CUELLO UTERINO Agosto 74 Junio 76 BIOPSIA

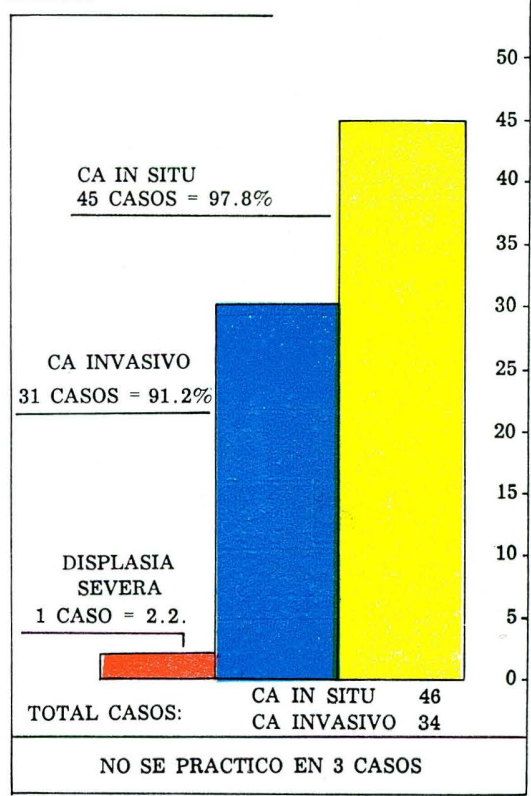

Gráfica Nọ 9

HOSPITAL FEDERICO LLERAS ACOSTA

CARCINOMA CUELLO UTERINO Agosto 74 - Junio 76 CONDUCTAS

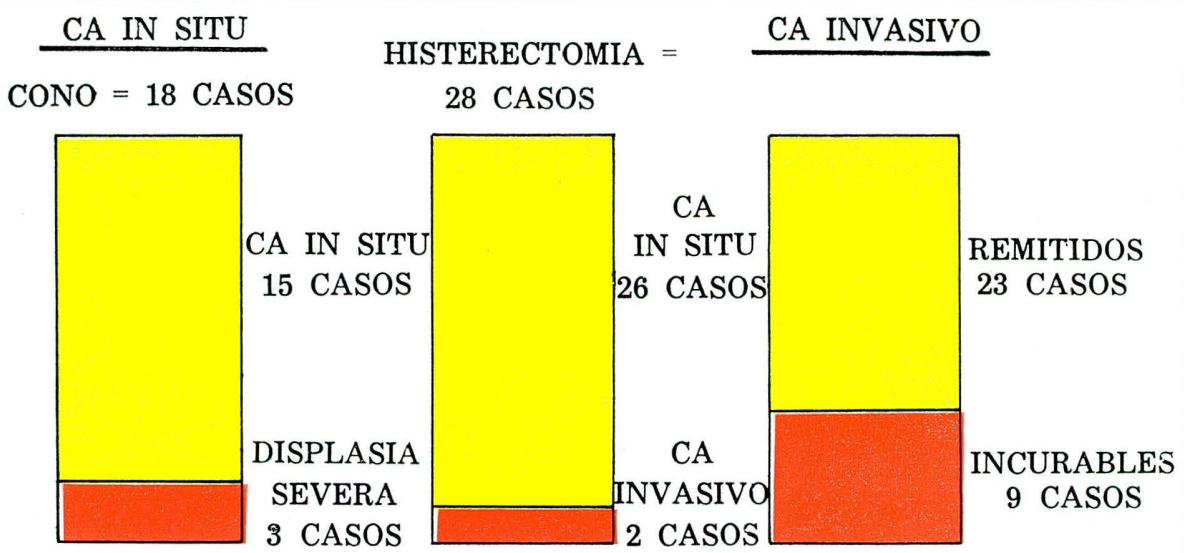


GRUPOS DE EDADES EN TRATAMIENTO (Gráfica No 10)

Gráfica Nọ 10

HOSPITAL FEDERICO LLERAS ACOSTA

CARCINOMA CUELLO UTERINO Agosto 74 - Junio 76

EDAD Y TRATAMIENTO

\begin{tabular}{|c|c|c|c|c|c|}
\hline \multicolumn{2}{|l|}{ CONO } & \multicolumn{3}{|c|}{$\begin{array}{c}\text { EN } 80 \text { CASOS } \\
\text { TRATADOS }\end{array}$} & \multirow[t]{2}{*}{ HISTERECTOMIA } \\
\hline & & 0 & $10-19$ & 0 & \\
\hline & $50 \%$ & 9 & $20-29$ & 2 & $7.1 \%$ \\
\hline & $44.4 \%$ & 8 & $30-39$ & 12 & $42.9 \%$ \\
\hline $5.6 \%$ & & 1 & $40-49$ & 10 & $35.7 \%$ \\
\hline & & & $50-59$ & 3 & $10.7 \%$ \\
\hline$\ldots \ldots$ & & & $60-69$ & 1 & $3.6 \%$ \\
\hline & & & $70-79$ & & \\
\hline & & 18 & TOTAL & 28 & \\
\hline
\end{tabular}

Podemos observar, que en el grupo de 20 a 29 se practicaron 9 conos y 2 histerectomías, En el grupo de 30 a 39,8 conos y 12 histerectomías en el grupo de 40 a 49,1 cono y 10 histerectomías, en grupos de mayores eda. des solamente se practicó histerectomía ampliada. 
PROCEDENCIA Y TRATAMIENTO

Gráfica Nọ 11

HOSPITAL FEDERICO LLERAS ACOSTA

CARCINOMA CUELLO UTERINO Agosto 74 - Junio 76

PROCEDENCIA Y TRATAMIENTO

\begin{tabular}{|l|r|r|c|r|r|r|r|}
\hline \multicolumn{2}{|l|}{} & PROCEDENCIA & \multicolumn{5}{|c|}{ HISTERECTOMIA } \\
\hline \multicolumn{2}{|l|}{ CONO } & $77.8 \%$ & 14 & RURAL & 18 & $64.2 \%$ & \\
\hline & $22.2 \%$ & 4 & URBANA & 10 & \multicolumn{3}{|c|}{$35.8 \%$} \\
\hline
\end{tabular}

TOTAL DE CASOS TRATADOS $=46$

Se practicaron 14 conos a pacientes del area rural y 4 a pacientes del area urbana, Histerectomía a 18 de area rural y 10 del area urbana.

MORBILIDAD (Gráfica 12)

Gráfica Nọ 12

HOSPITAL FEDERICO LLERAS ACOSTA

CARCINOMA CUELLO UTERINO Agosto 74 - Junio $76 \hat{~}$ MORBILIDAD

\begin{tabular}{|c|c|c|}
\hline \multicolumn{3}{|l|}{} \\
\hline ABSCESO DE PARED & 1 & $2.2 \%$ \\
\hline HEMATOMA CUPULA & 6 & $13 \%$ \\
\hline NINGUNA & 39 & $84.8 \%$ \\
\hline
\end{tabular}

Encontramos 1 absceso de pared y 6 hematomas de cúpula para un

\section{RESUMEN Y CONCLUSIONES}

Se estudian 80 enfermas vistas du. rante dos años en el Hospital Regio-
$15,2 \%$, de morbilidad. No hubo mortalidad.

nal Federico Lleras, de Ibagué clasificadas con diagnóstico de carcinoma del cuello uterino, 46 in situ y 36 invasivo. La mayor incidencia del carci- 
noma in situ fue de la tercera década, en cambio en el carcinoma invasivo fue la cuarta década.

No se encontró en nulíparas. En primíparas solamente un caso de carcinoma invasivo. En cambio, para ambos grupos fue muy alta la incidencia en grandes multíparas.

En relación con la zona, es mayor los casos de la urbana, pero se aprecia positivamente, un buen número de casos de área rural con carcinoma in situ, que han podido ser diagnosticados por controles citológicos hechos en puestos de salud y remitidos al Hospital. El aspecto clínico en el carcinoma in situ no muestra lesiones sospechosas ni tumorales, un gran porcentaje es de apariencia sana. En tanto en el invasivo se le encuen. tra reportado en gran mayoría como tumoral. Los dos casos reportados co. mo sano fueron dos microinvasivos. La citología se practicó en 42 casos, confirmándose en 39 carcinoma in situ.

Las biopsias mostraron carcinoma in situ en 45 casos y displasia severa en 1.

Se practicaron 28 histerectomías $60 \%$ y $18 \%$ conizaciones $40 \%$. Creemos que debe limitarse cada vez más la conización y pasar a la etapa de histerectomía, limitando la primera a pacientes jóvenes, que deseen tener más hijos, de difícil seguimiento 0 que rehusen la histerectomía. carcinoma in situ was found during the third decade, while for tre invasive carcinoma the greatest incidence was found in the fourth decade.

It was not found in nulliparous. In primiparous only one case of invasive carcinoma was found and for multiparous the incidence was very high in the two groups.

In what refers to the area, most of the cases were found in the urban zone, but a good number of the significant cases with carcinoma in situ detected in the rural area could have been diagnosed by cytologicai controls in health posts and then referred to the hospital.

The clinical aspect in the carcinoma in situ does not show suspicious or tumoral injuries,a great percentage of themis of healthy appearance. Mean while a great mayority of the invasive carcinoma has been reported as tumoral. The two reported healthy cases were microinvasive. Citology was practiced in 42 cases, retifying thus 39 carcinomas in situ cases.

The biopsies done showed carcino. mia in situ in 45 cases and severe dysplasia in one patient.

\section{CERVIX CARCINOMA}

\section{SUMMARY AND CONCLUSIONS.}

The study covers eighty patients followed up during two years at the Hospital Regional Federico Lleras, of Ibagué, classified with a diagnosis of Cervix Carcinoma of Which there were 46 in situ cases and 36 invasive cases, The greatest incidence of the 


\section{CARCINOMA DEL CUELLO UTERINO BIBLIOGRAFIA}

1. BOTELLA LLUSIA. Enfermedades del aparato genital femenino Tomo II. Págiria 467 y siguientes.

2. BEDOYA MARIANO. y Col. EpidemioIcgía del Cáncer del Cérvix en êl Perú Revista Colombiana de Obstetricia y Ginecología. Vol. XXIII. № 5 septiembre - octubre 1972.

3. ERAZO JOSE VICENTE. y Col. Variables epidemiclógicas relacionadas con el Cáncer del cuello uterino en pacientes con citologías III. IV y V. eri Po- payán 1971. Revista Colombiana de Obstetricia y Ginecología. Vol. XXIII. № 6 noviembre - diciembre 1972.

4. FONNEGRA ALVARO. y Col. Relación entre Displasia severa carcinoma in situ del cuello uterino. Revista Colom biana de Obstetricia y Giriecología. Vol XXIV. N: 5. septiembre - octubre 1973.

5. JORDAN GERMAN y Col. Avances en el manejo de las neoplasias intraepiteliales. Boletín del Instituto Nacional de Cancerología. Vol 2 №5. julio diciembre 1973. 